\title{
Resources review
}

\section{The Teamwork File: National Cancer Alliance 2003}

\section{Heather Goodare MA Dip. Couns.}

3 Glengyle Terrace, Edinburgh EH3 9LL, UK.

E-mail: hm.goodare@virgin.net

Patient-held records have long been standard in maternity care, but are a relatively new concept in cancer care, perhaps because for too long patients were not told the truth, or at any rate the whole truth, about their disease. Things are now changing.

The National Cancer Alliance (NCA), which is a UK association of patients, carers, and healthcare professionals, has led the way in piloting such a project in the cancer field, and the present document is the second edition (2003) of their originally somewhat unwieldy 'Teamwork File'. This now consists of two separate booklets, an A5 ring binder to hold a 'personal health record', and an A4 'Guide to Cancer', giving general cancer information. The two documents are presented in a stout A4 plastic wallet, together with a publicity leaflet.

A great deal of work has obviously gone into the production of this material, which has been extensively researched and piloted. ${ }^{1}$ The idea that patients and professionals, or 'users' and 'providers' of services, should work together as a team can only be applauded. However, to some extent this initiative has been overtaken by others, no doubt inspired by its example. As the Teamwork authors acknowledge, 'More and more, hospitals are introducing "patient-held records", and it is interesting to compare one of these, the Sussex Patient Held Record, last revised in August 2004, with the 'Personal Health Record' provided by the Teamwork File.

They share many features: spaces for writing down contact numbers, details of medication, chemotherapy and radiotherapy appointments and protocols, and personal comments and questions. Both are in A5 size, the NCA file in portrait format and the Sussex in landscape. The Sussex file also gives basic cancer information, for carers as well as patients, in addition to contact numbers for national support organizations and details of further reference material. It has been developed by healthcare professionals, and while not being patient-unfriendly has a business-like style that seems to encourage use by professionals as well as patients. Its aim is stated as: 'To help improve information to Patients and communication between Hospital Staff, General Practitioners, District Nurses, and other Community Staff.'

The NCA file provides general cancer information in its own separate A4 66-page booklet, The Teamwork Guide to Cancer. This is lavishly produced in two colours, with (in my view) unnecessarily large type and wide margins: while much of it is of permanent relevance, the lengthy 18-page section of 'Useful Contacts' is already out of date. The booklet has been approved by the Plain English Campaign, and is clearly written, but explanations are sometimes so oversimplified as to be misleading - for instance the definition of 'cervix': 'Part of a woman's body found under the womb and above the vagina'. Also it is a pity to see a few obvious misprints in a second edition. 
The question of format for such productions is crucial. A4 booklets without a spine tend to get lost on a shelf, and it would be more practical, I suggest, to produce a smaller, thicker booklet giving general information, which would fit better on to an ordinary bookshelf together with the personal health record, and be easier to carry around. The type size could easily be reduced and some of the information pruned. Many of the contact details for support organizations, which date quickly, could be cut, and patients directed to the main umbrella organizations instead. A very useful 64-page booklet could still be published, though whether it would replace the Macmillan Cancer Guide is a moot point.

It seems helpful to compare the Teamwork Guide to Cancer with the Macmillan Cancer Guide, as this is recommended as a source of further information in the Sussex Patient Held Record, and is freely available (from Macmillan Cancer Relief, Tel.: 0808808 2020). The current Macmillan Guide (first published in 1997 by BBC Educational Developments) is dated 2004, and its format is A5; it contains 56 pages, with a further six-page leaflet on Living with cancer: what you can expect from the NHS bound into the centre fold. It is printed in full colour, with some appealing photographic illustrations, and has much the same sections and information as the Teamwork guide, but in my view is handier to use as it can be more easily popped into a handbag or briefcase, and will even slot inside the Sussex Patient Held Record. Its glossary is shorter (85 entries as opposed to 145) but includes some terms that the Teamwork guide omits, such as 'alopecia' and 'histology'. Its list of helpful organizations is also shorter, but more up-to-date, and again includes bodies omitted by the Teamwork guide for instance, Radiotherapy
Action Group Exposure (RAGE), for women permanently damaged by radiotherapy for breast cancer.

To sum up, this project has been inspirational and trail-blazing, but possibly over-ambitious and lavish in terms of production. The time may now have come for a national organization, perhaps the NCA itself, to survey all the various examples of similar projects from hospitals around the country and produce yet another model version of the patient-held record for people with cancer that can be adopted by the NHS nationwide. It is certainly time for it, as cancer patients, perhaps more than most, have to travel between hospitals for their various treatments and need to have their personal record to hand, not only so that they can keep track of their own progress, but also to have details of their current treatment readily available if their hospital records go astray - which is by no means unheard of. Centralized electronic records may help, but will not replace such personally held information. It is important too that healthcare professionals routinely fill in the required details in the patient's file, and this may necessitate some staff training to overcome paternalistic attitudes.

In the meantime, any patient may contact the NCA for a free copy of the file, by telephoning 01865225294 or by accessing their website at http://www.teamworkfile.org.uk. A video is available for South Asian patients (not reviewed here).

\section{Reference}

1 Smith C. The role of health professionals in informing cancer patients: findings from The Teamwork Project (phase one). Health Expectations, 2000; 3: 217-219. 